\title{
The issue of loading the material in a vibro- impact grinder
}

\author{
Olena Fedoskina ${ }^{1}$, Valerii Fedoskin ${ }^{1, *}$, and Anastasiia Loginova ${ }^{2}$ \\ ${ }^{1}$ National Technical University Dnipro Polytechnic, 49005, Dnipro, Dmytra Yavornytskoho Ave., 19, \\ Ukraine \\ ${ }^{2}$ Institute of Geotechnical Mechanics named by N. Poljakov of National Academy of Sciences of \\ Ukraine, 49005, Dnipro, Simferopolska Str., 2a, Ukraine
}

\begin{abstract}
Absrtact. The article discusses the basic principles of force loading of a material in a vibro-impact grinder with a vertical and inclined working chamber. It is shown that in a grinder with a vertical working chamber the control of the material loading is limited by the presence of a relationship between the vertical and rotational oscillations of the jaws. Vibro-impact grinder with an inclined working chamber represents a new class of machines. A mathematical model of the process of interaction of the jaw with a piece of material when moving it in the working chamber is presented. Equations and graphical dependencies are obtained, which determine the phase angle of the moment of clamping the piece in the working chamber. The modes of power loading of the material in the working chamber were analyzed.
\end{abstract}

\section{Introduction}

In modern technological processes, the material grinding is widely used in the preparation, in the intermediate and final stages of the finished product. Making a fine-grained and powder product from strong and abrasive materials using crushing and reducing equipment is associated with considerable difficulties. Crushing strong materials in the jaw and roll crushers by crushing is characterized by low speed and the number of power effects on the piece during the passage of the working chamber. As a consequence of this we have a small (3-5) degree of crushing and high power intensity of obtaining a fine-grained product. Crushing and grinding equipment with a dynamic method of material loading has a number of significant defects.

The processing of strong and abrasive materials in hammer and rotary crushers leads to the intense wear of the working surfaces of the executive body; disintegrators in addition to wear are limited by the size of the input fraction; drum ball mills have low efficiency and ineffective in light manufacturing. Vibratory mills and cone inertial crushers have good performance in the processing of strong materials, however, with an increase in their size, the grinding efficiency decreases, which requires preliminary preparation of the input fraction.

${ }^{*}$ Corresponding author: fedoskin_va@ukr.net 
Vibration jaw grinders are the machines with a dynamic method of impact on the material. Realized in these grinders high-frequency shock loading of the material leads to the opening of internal cracks, the accumulation of residual deformations and the reduction of destructive stresses.

The increasing number of productions that use equipment with a capacity from kilograms to several tons per hour, toughening the requirements for the particle size distribution of the grinded product, and the form of the resulting grain, puts forward the task of researching existing grinders and creating new ones with adjustable parameters that form the required force impact on material.

\section{Method}

Research conducted at the National Technical University "Dnipro Polytechnic" for the first time proved the possibility of using a vibration jaw grinder with a pendulum jaw suspension as an independent grinding unit for producing fine-grained and powder materials [1].

A vibro-impact grinder with a vertical working chamber (Fig. 1) consists from jaws (1) suspended by axes (2) to body (3), which is supported by springs (4) on the base. A pendulum vibration exciter (6) with an unbalance shaft (7) is fixed on each jaw. The longitudinal axis (9) of the pendulum (6) makes an angle $\zeta$ with the horizontal plane (10). At the neutral position, the jaws are held by elastic elements (11). Vibrating exciter (6) is connected to the jaw (1) by elastic elements (8). This reduces the shock loads on the bearing units while retaining all the features of the grinder. Synchronous antiphase movement of the cheeks is provided by vibration exciters (6) by mechanical synchronizers or using the effect of self-synchronization.

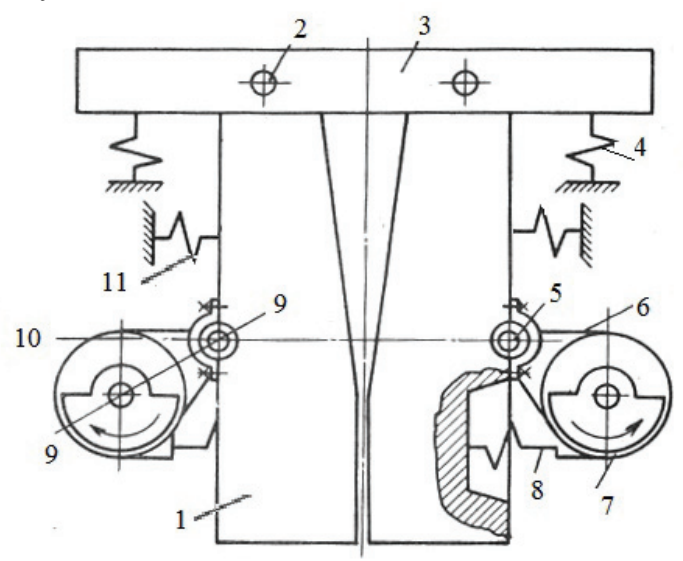

Fig. 1. The constructive scheme of a vibro-impact grinder with a vertical working chamber.

In this grinder design, there are two oscillatory circuits: the first one consists from the jaw, elastic elements, single-shaft vibration exciter; the second one consists from a grinder body with jaws, shock absorbers, a self-balanced vibration exciter (formed by vibration exciters of the jaws). The first circuit provides rotary vibrations of the jaws and the force load on the material, the second one provides vertical vibrations of the grinder, affecting the speed of material movement relative to the jaws.

Despite the interrelation of vertical and rotational oscillations, this system makes it possible, within certain limits, to regulate the magnitude of the force effect on the material and the number of impacts during the time of passage of the working chamber. 
The value of the shock pulse is controlled by changing the angle of inclination of the axis of the pendulum. When the inclination of axes (9) is $\zeta=0^{\circ}$, there are practically no vertical oscillations of the body, and the shock pulse has a maximum value, and the velocity of the relative movement of the material is minimal. Increasing angle $\zeta>0^{\circ}$ leads to the decreasing of the shock pulse magnitude with increasing velocity of the material.

The speed of the material when saving the power parameters can be reduced by rotating the unbalance shafts counterclockwise (opposite to the rotation indicated in Fig. 1). In this mode, the time spent by the substandard product in the working chamber increases due to the convergence of the jaws during the upward movement of the body (against the movement of material flow).

Despite the high frequency of jaws oscillation, the number of blows inflicted on the proceeding material during the time of passage of the working chamber is often not sufficient for the effective destruction of particularly durable natural and artificial materials, which is associated with a mismatch between the frequency and speed of the material. As a consequence, we have an increasing circulation load under operating in a closed cycle, as well as the need to organize several grinding stages, which leads to high energy costs, increased metal consumption, and increased space occupied by the equipment. In addition, there are a number of materials that require a strictly metered load from the jaws.

\section{Results}

A vibro-impact grinder (Fig. 2) with an inclined working chamber solves the task of independently changing the shock pulse magnitude, the frequency of oscillation of the jaws and the speed of the material. In general, the grinder contains an active (upper) jaw (3) pivotally mounted in the body (passive jaw) (1) with lining (7). The active jaw is equipped with a two-shaft inertial exciter (4) and is connected by an elastic element (6) with the grinder body, which is installed by f shock absorbers (5) to the base.

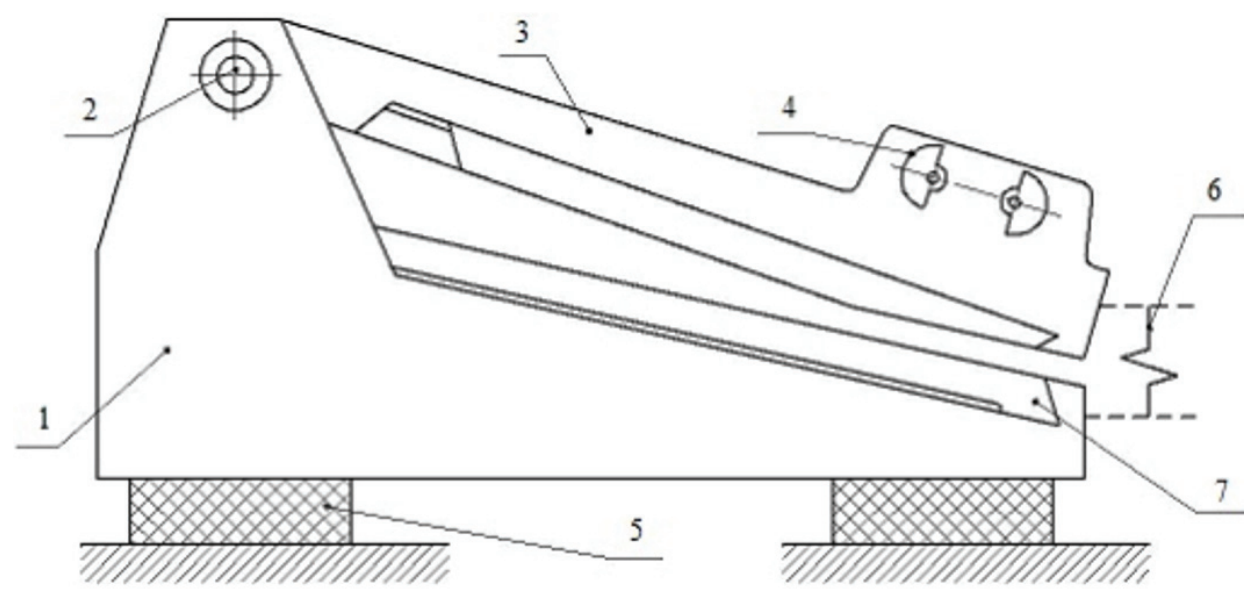

Fig. 2. The constructive scheme of a vibro-impact grinder with an inclined working chamber.

The material moves to the discharge window due to the inclination angle of the working surface of the passive jaw and its oscillations under the perturbing force of the vibration exciter (4). In this case, the amplitude of the body oscillations is much less than the amplitude of the active jaw oscillations. Placing the vibration exciter on the active jaw provides for the required shock pulse almost independently of the material speed, which makes it possible to vary the parameters and the force effect on the material over a wide 
range, expands the area of control of the grinding process and implement specific technological modes.

When considering the movement of a piece of material (particle), subjected to clamping (destruction) between the two surfaces of the working chamber, we assume that the passive jaw is fixed, and the material moves at an average speed:

$$
V=f(S, \omega)
$$

where $S$ - a distance, passing by the piece of material for the period.

The active cheek performs harmonic oscillations according to the law $X=A \sin (\omega t)$.

The calculated scheme of interaction of a piece of material (particle) with the working surfaces of the chamber is shown in Figure 3.

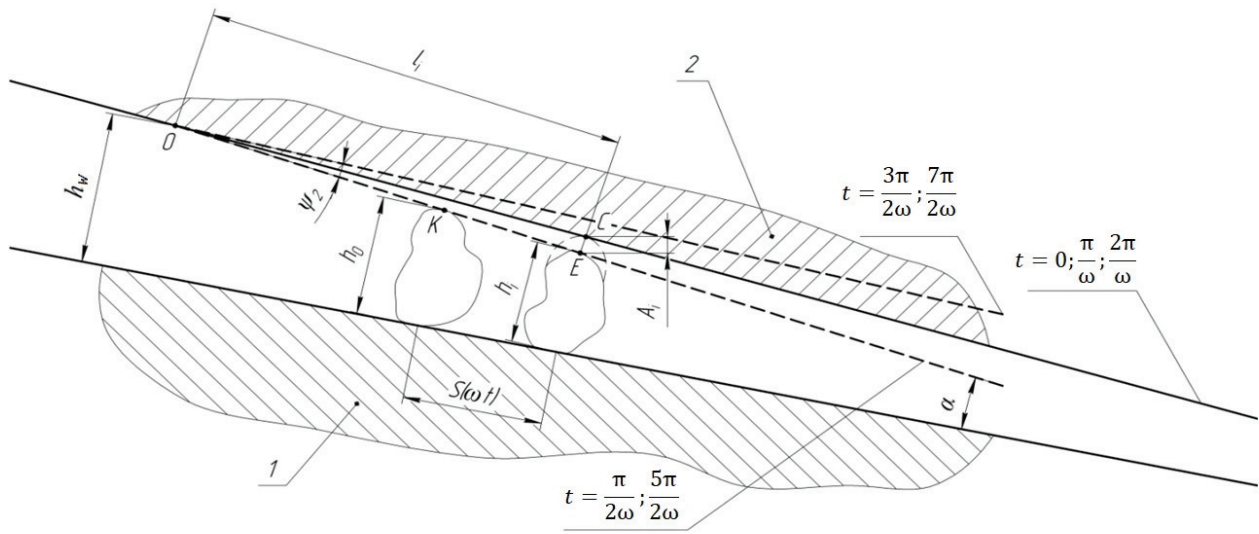

Fig. 3. The calculated scheme of interaction of a piece of material (particle) with the working surfaces.

The scheme shows: 1 is active jaw; 2 is passive jaw; $O$ is the axis of suspension of the active jaw; $h_{w}$ is the height of the boot window of the grinder; $h_{0}$ is the transverse size of the original piece of material; $h_{i}$ is the transverse size of a piece of material in the $i$-th section of the working chamber; $l_{i}$ is the distance from the axis of suspension to the $i$-th section of the working chamber; $S(\omega t)$ is the distance covered by the piece of material; $\psi_{2}$ is the angle of rotation of the active cheek relative to the axis of suspension, $\alpha$ is the angle of capture, $A_{i}$ is the amplitude of the relative oscillations of the jaw in the $i$-th section of the working chamber.

A piece of material with a cross section of size $h_{0}$ enters the loading area, touches the working surface of the active jaw in its lowest position (point $K$ ). At this moment, the speed of the piece has a zero value, the jaw begins to move to the highest position, the contact of the piece with the jaw disappears and its movement to the discharge window resumes.

The next contact of the piece with the jaw occurs in the $i$-th section of the working chamber (point $C$ ). Because the point of contact is located on the path of movement of the jaw, the piece is deformed and destructed. The extreme lower position of the jaw characterizes the end of the process of destruction (deformation) of a piece of material (point $E$ ), the beginning of the jaw movement up and the appearance of a gap between the surface of the jaw and the material.

The transverse size of the piece, after interacting with the jaw in the $i$-th section of the working chamber, is:

$$
h_{i}=h_{w}-l_{i}\left(\tan \frac{\psi_{2}}{2} \cos \alpha+\tan \alpha\right)
$$


Jaw oscillation amplitude in the $i$-th section of the working chamber is:

$$
A_{i}=l_{i} \tan \frac{\psi_{2}}{2} \cos \alpha
$$

The gap between the piece of material and the surface of the active jaw when it departs in the $i$-th section of the working chamber is $\Delta\left(A_{i}\right)=A_{i}-A_{i} \sin \omega t$.

This gap, in the presence of the angle of capture, decreases in the process of movement of the piece to the discharge window by $\Delta(S)=S(\omega t) \cdot \tan \alpha$.

Then, the current gap between the piece of material and the surface of the active jaw in the $i$-th section of the working chamber is:

$$
\delta_{i}(\omega t)=A_{i}-A_{i} \sin \omega t-S(\omega t) \tan \alpha
$$

The phase angle $\varphi_{k}=\omega t_{k}$, which determines the beginning of the moment of clamping $t_{\mathrm{k}}$ of the piece in the working chamber, corresponds to the equality to zero of the current gap and is found from the equation:

$$
A_{i}-A_{i} \sin \varphi_{k}-S\left(\varphi_{k}\right) \tan \alpha=0
$$

Figure 4 presents the graphical dependence of the phase angle of the clamping of the material. Curve 1 describes the change in the gap when moving the jaws, curves 2, 3, 4 characterize the change in the gap when moving a piece of material.

The abscissa of the curves intersection point determines the value of the phase angle $\left(\varphi_{k}=\omega t_{k}\right)$ where the gap between the material and the surfaces of the cheeks is zero. In the presented graph, the intersection of curves 1 and 2 (point $C$ ) corresponds to the phase angle $\varphi_{k}=2 \pi$. This is the most effective mode of material loading in the $i$-th section of the working chamber where the jaw has the maximum impact velocity at the moment of contact with the piece, and the piece receives a deformation equal to the amplitude of the oscillations of the jaw. At the end of the material clamping (point $E$ ) and the beginning of a new cycle, the lateral dimension of the piece is equal to $h_{i}$ (Fig. 3). In the process of following movement of the piece to the discharge window, several modes of its clamping in the working chamber are possible.

Mode 1. Under the condition $h_{i+1}+A_{i+1}=h_{i}$, the phase angle, which determines the moment when the piece contact the jaw in the $i+1$ section of the working chamber, is also equal to $\varphi_{k}=2 \pi$. This ensures the greatest efficiency of material destruction.

Mode 2. The inequality $h_{i+1}+A_{i+1}<h_{i}$ determines the contact of a piece of material with the jaw in $i+1$ cross section of the working chamber above the equilibrium position. The phase angle determined by the intersection of curves 1 and 4 has the values of $3 / 2 \pi<\varphi_{k}<2 \pi$. This leads to a decrease in the impact speed and the efficiency of grinding the material. If in one period the lateral dimension of the piece does not change, then in subsequent periods the phase angle of the clamping will decrease and, if $\varphi_{k}=3 / 2 \pi$, the jaws become wedged.

Mode 3. When $h_{i+1}+A_{i+1}>h_{i}$, the piece of material contacts with the jaw in $i+1$ section of the working chamber below the equilibrium position. The phase angle determined by the intersection of curves 1 and 3 has the values $2 \pi<\varphi_{k}<5 / 2 \pi$.

When the phase angle of the clamping of the piece is increased, the values of impact jaw speed, deformation of the piece, as well as the duration of the clamping are decreased and at $\varphi_{k}=5 / 2 \pi$ are equal to zero. Similar to mode 2 , if in one period the lateral dimension of the piece does not change, then in subsequent periods the phase angle of the clamping of the piece will decrease. 


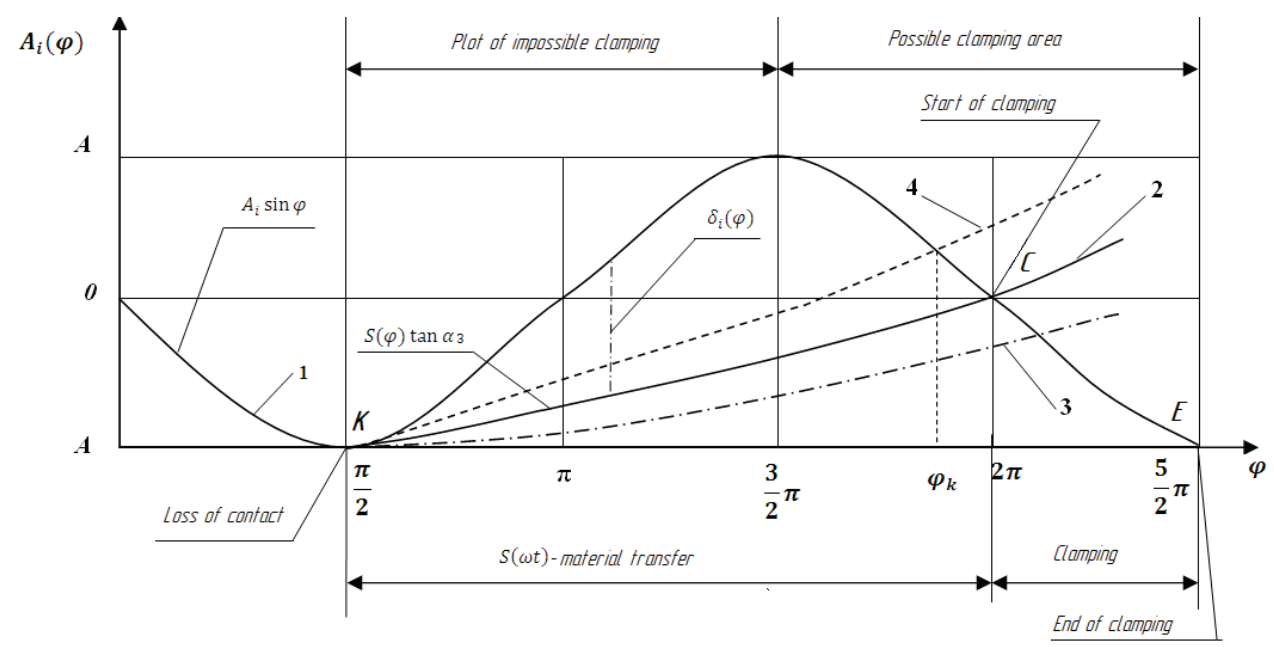

Fig. 4. Graphic dependence of the phase angle of material clamping.

However, in this mode, as the phase angle decreases to $\varphi_{k}=2 \pi$, the impact jaw speed increases with each subsequent period, that leads to increasing the force effect on the material. If a piece of material does not collapse under the phase angle $\varphi_{k}=2 \pi$, and material continues to move to the discharge window, then subsequent periods correspond to Mode 2, which is characteristic of passing through the working chamber of an undivisible body.

\section{Conclusions}

The mathematical model makes it possible to obtain new dependencies that describe the interaction of the jaws with the material and to determine the rational mode of force loading. The vibro-impact grinder with an inclined working chamber, a pendulum suspension of one active jaw, equipped with a two-shaft inertial vibration exciter, is a new class of crushing and grinding machines, which allow independently controlling the dynamic process of power loading of the material and its speed in the working chamber over wide limits.

\section{References}

1. Franchuk V.P., Fedoskin V.A., Plakhotnik V.V. (1990). Prospects for the use of vibratory jaw crushers for grinding ferromaterials. Obogashcheniye poleznykh iskopayemykh, (40), 3-6

2. Franchuk V.P., Fedoskina O.V. (2008). Vibracijna shchokova drobarka Patent No 89439, Ukraine. 